\title{
Diagnostic Value of Endomyocardial Biopsies of the Right Ventricular Septum in Arrhythmias Originating from the Right Ventricle
}

\author{
Stefan Peters,* MD, Michael J. Davies, MD, \\ and William J. McKenNA, MD
}

\section{SUMMARY}

The aim of the study was to classify the value of endomyocardial biopsies taken from the right ventricular septum in patients with ventricular tachyarrhythmias originating from the right ventricle.

In a cohort of 62 young patients (mean age 35.6 years) 4-5 endomyocardial biopsies were taken from the right ventricular septum and myocardial atrophy, fibrosis and lipomatosis were analysed qualitatively and semi-quantitatively. Much interest was focused on the arrangement and type of fibrosis. Lipomatosis, myocardial atrophy and a finer form of fibrosis surrounding individual myocytes or groups of myocytes were judged as the typical histopathological findings of arrhythmogenic right ventricular dysplasia.

The cohort of patients was subdivided clinically into idiopathic right ventricular outflow tract tachycardia $(n=50)$ and arrhythmogenic right ventricular dysplasia $(n=12)$.

In the group of arrhythmogenic right ventricular dysplasia pathological findings were found in $92 \%$ with typical fibrolipomatosis in two cases, severe fibrosis ( $>40 \%$ per biopsy) in 5 cases, a finer form of fibrosis surrounding individual myocytes without lipomatosis in two cases and a slight interstitial or subendocardial fibrosis in two cases. In only one patient the endomyocardial biopsy was normal.

In right ventricular outflow tract tachycardia normal endomyocardial biopsy findings predominated $(80 \%)$. In two cases lipomatosis without fibrosis was a more or less normal finding, and in two other cases a slight interstitial or subendocardial fibrosis was found. Fibrosis was judged to be moderate or severe with discrete signs of inflammation in only two cases; in a long-term follow-up these two patients developed typical features of idiopathic dilated cardiomyopathy.

Endomyocardial biopsies taken exclusively from the right ventricular septum were able to distinguish between right ventricular outflow tract tachycardia and arrhythmogenic right ventricular dysplasia, although the typical finding of

\footnotetext{
From the *University Hospital Magdeburg, Magdeburg, Germany, and the Department of Cardiological Sciences, St. George's Hospital Medical School, London, UK.

Address for correspondence: Stefan Peters, MD, University Hospital Magdeburg, Cardiology, Leipziger Str. 44, 39120 Magdeburg, Germany.

Received for publication Septenber 26, 1995.

Accepted October 25, 1995.
} 
fibrolipomatosis in cases with arrhythmogenic right ventricular dysplasia was rare. Characteristics of fibrosis are the key to the correct diagnosis provided that histopathological analysis is based on experienced qualitative criteria (type and arrangement of fibrosis).

In conclusion, qualitative and semi-quantitative analyses of endomyocardial biopsies are far better than computerized quantitative methods. Biopsies from the free right ventricular wall cannot be generally recommended if a correct definition of arrhythmoge nic right ventricular dysplasia in endomyocardial biopsies from the right ventricular septum is used. (Jpn Heart J 1996; 37: 195-202)

Key words: Endomyocardial biopsy Fibrosis Arrhythmogenic right ventricular dysplasia Right ventricular outflow tract tachycardia

\begin{abstract}
A RRTHYMIAS originating from the right ventricle are one of the most important forms of nonischemic tachyarrhythmias. Some forms of right ventricular arrhythmias have their origin in the right ventricular outflow tract with morphological changes not yet well defined. Other forms of arrhythmias belong to a cardiac entity called 'arrhythmogenic right ventricular dysplasia' or 'cardiomyopathy' with muscular atrophy of the right ventricle and with striking fibrolipomatosis which can be best demonstrated by autopsy and target-directed intraoperative biopsy. The term 'fibrolipomatosis' is characterized by a predominant lipomatosis and a finer form of fibrosis surrounding individual myocytes or groups of myocytes."

The value of percutaneous endomyocardial biopsy taken from the right ventricular septum in arrhythmogenic right ventricular dysplasia is generally limited by sparing of the septum and of subendocardial areas in many cases. ${ }^{2)}$

Other histological definitions of arrhythmogenic right ventricular dysplasia include a striking fibrosis of more than $40 \%$ in biopsy specimens taken from the right ventricular septum ${ }^{3)}$ or different forms of fibrosis (focal, interstitial and subendocardial) in the same biopsy ${ }^{4)}$. The goal was to clarify the role of classical endomyocardial biopsy taken from the right ventricular septum with a low, acceptable complication rate in a cohort of patients with arrhythmias originating from the right ventricle. The question remains whether biopsies from the free right ventricular wall should be generally recommended despite a probably higher complication rate.
\end{abstract}

\title{
Methods
}

Complete invasive examination, including endomyocardial biopsy, was conducted in 29 females and 33 males (mean age $35.6 \pm 13.4$ years) with arrhythmias originating from the right ventricle documented by electrophysiological studies. 
Four to five endomyocardial biopsies were taken from the right ventricular septum in each patient by a King bioptome via the vena jugularis or by modified King bioptome via the vena femoralis.

Light microspcoic cxamination was done in the Department of Pathology (Prof. MJ Davies) without knowledge of the clinical diagnosis. The following parameters were cxamined: mild $(+)$, moderate $(++)$ and severe $(+++)$ fibrosis, lipomatosis without fibrosis, typical fibrolipomatosis (severe lipomatosis with a finer form of fibrosis), subendocardial fibrosis, inflammation and the form of fibrosis (subendocardial, focal, interstitial or surrounding individual myocytes or groups of myocytes). Severe fibrosis usually means $40 \%$ fibrotic material per biopsy specimen. In all patients left and right ventricular angiography, coronary angiography, standard ECG, signal-averaged ECG and transthoracic echocardiography were performed; arrhythmogenic right ventricular dysplasia was diagnosed clinically with regard to previously published major and minor criteria. ${ }^{12)}$

Nearly all patients were re-examined clinically at regular follow-up of at least 2 years.

\section{Results}

Endomyocardial biopsy was normal in 41 patients with right ventricular arrhythmias. Fibrolipomatosis - the typical histological finding of arrhythmogenic right ventricular dysplasia - could be demonstrated in only two cases. Severe fibrosis $(+++)$ was found in 6 patients: in two cases with inflammation, in one as a focal and interstitial form, in two as a focal fibrosis associated with other forms of fibrosis and in the last as a severe form of fibrosis surrounding individual myocytes. Subendocardial fibrosis as the only pathological finding was found in two cases.

A moderate form of fibrosis (+t) was the finding in one case with a small amount of inflammation. Mild fibrosis $(+)$ was the result of endomyocardial biopsies in 8 cases: surrounding individual myocytes or groups of myocytes in two and interstitial forms in 6 . Lipomatosis without fibrosis could be demonstrated in two

Table I. Endomyocardial Biopsy in Right Ventricular Outflow Tract Tachycardia

\begin{tabular}{lc}
\hline Finding & Normal \\
\hline Slight subendocardial fibrosis & 40 \\
Slight interstitial fibrosis & 1 \\
Severe fibrosis with inflammation & 5 \\
Lipomatosis without fibrosis & 1 \\
Number of cases & 2 \\
\hline
\end{tabular}


Table II. Endomyocardial Biopsy in Arrhythmogenic Right Ventricular Dysplasia

\begin{tabular}{lc}
\hline Finding & Number of cases \\
\hline Typical fibrolipomatosis & 2 \\
Finer surrounding fibrosis & 2 \\
Severe surrounding fibrosis & 1 \\
Focal fibrosis (+++), slight surrounding fibrosis & 1 \\
Severe focal and interstitial fibrosis & 1 \\
Severe interstitial fibrosis with inflammation & 1 \\
Severe focal replacement fibrosis & 1 \\
Slight interstitial fibrosis & 1 \\
Slight subendocardial fibrosis & 1 \\
Normal & 1 \\
\hline
\end{tabular}

cases.

With regard to clinical aspects, two major or one major and two minor criteria of arrhythmogenic right ventricular dysplasia with localized right precordial QRS prolongation, positive late potentials, isolated dilatation of the right ventricular outflow or inflow tract or angiographic abnormalities, i.e. enddiastolic-endsystolic bulging with loss of trabecular structure were observed in 12 patients; in this group the diagnosis of arrhythmogenic right ventricular dysplasia was defined clinically.

The other group of 50 patients was defined as having idiopathic right ventricular outflow tract arrhythmias.

The value of the endomyocardial biopsy was redefined after clinical definition of arrhythmogenic right ventricular dysplasia and idiopathic right ventricular outflow tract arrhythmias. In the group of 50 patients with so-called idiopathic right ventricular outflow tract arrhythmias 40 had normal findings in 4-5 biopsies taken from the right ventricular septum. A mild subendocardial fibrosis appeared in one patient, and mild interstitial fibrosis in 5. Lipomatosis without pathological fibrosis or myocyte abnormalities belonged to the spectrum of normal findings in two female patients. In only two patients moderate or severe fibrosis with associated mild inflammation could be found (Table I). In a followup of at least two years these two patients developed typical clinical symptoms and findings of idiopathic dilated cardiomyopathy with progressive left ventricular dilatation. In the other 48 patients, arrhythmias were controlled with antiarrhythmic drugs, or in a few cases, by catheter ablation.

Diagnosis could be confirmed by the typical pattern of fibrolipomatosis in endomyocardial biopsy in the group of patients with arrhythmogenic right ventricular dysplasia. In two other cases the typical finer form of fibrosis surrounding individual myocytes or groups of myocytes was the only pathological finding. Severe forms of fibrosis such as replacement type, surrounding form of fibrosis or interstitial fibrosis with mild inflammation were found in five cases. In most cases 
with severe fibrosis ( $>40 \%$ per biopsy specimen) different types of fibrosis could be seen in the same biopsy. Only a mild interstitial or subendocardial form of fibrosis could be demonstrated in two cases of arrhythmogenic right ventricular dysplasia. Completely normal findings could be assessed in only one patient (Table II). During the follow-up period two patients died from sudden cardiac death despite well-controlled and serial-tested antiarrhythmic therapy, and two paticnts were treated by an automatic intrathoracic cardioverter-defibrillator because of refractory ventricular tachyarrhythmias and recurrent syncopes.

In arrhythmogenic right ventricular dysplasia pathological findings were present in $92 \%$, whereas in idiopathic right ventricular outflow tract arrhythmias normal findings predominated in $80 \%$.

\section{Discussion}

Endomyocardial biopsy is a well-accepted diagnostic method in a number of cardiac entities. The value of endomyocardial biopsy is well-established in graft rejection after heart transplantation and in infiltrative heart muscle diseases. In cardiac amyloidosis, for example, endomyocardial biopsy has a sensitivity of $100 \%{ }^{5)}$

The use of endomyocardial biopsy is still under debate in different types of so-called idiopathic cardiomyopathies and in arrhythmias without myocardial disease.

One of the most interesting groups of arrhythmias is nonischemic arrhythmias originating from the right ventricle. In many cases the right ventricular outflow tract is the origin of the arrhythmias. Structural abnormalities of this portion of the right ventricle could be demonstrated by angiography and a magnetic resonance technique. ${ }^{6)}$ Nevertheless, in most cases the ultrastructural cause of arrhythmias is not clear.

In a definite number of other cases the origin of the arrhythmias is another part of the right ventricle, i.e. the apical and inferior regions. The underlying morphology is fibrolipomatosis of the so-called triangle of the right ventricle of the infundibulum, the apex and right ventricular inflow tract. ${ }^{7)}$ These structural changes are summarized under the term 'arrhythmogenic right ventricular dysplasia'8) or 'arrhythmogenic right ventricular cardiomyopathy' ${ }^{9}$ ')

Endomyocardial biopsy can underline the diagnosis of arrhythmogenic right ventricular dysplasia but in many cases biopsy fails. This is particularly due to the fact that fibrolipomatosis is a process progressing from the subepicardial layers to intramural areas, sparing the left ventricle and right ventricular septum in most cases.

If biopsies are taken exclusively from the right ventricular septum 
pathognomonic fibrolipomatosis can be diagnosed in 10-20\%. The much higher frequency of typical findings in arrhythmogenic right ventricular dysplasia is in most cases based on the fact that biopsies were taken from the septoapical part of the right ventricle. ${ }^{3)}$ The working group of the University of Münster, Germany, started to take angiography-directed biopsies from areas of the right ventricular free wall with striking bulging or other structural changes. This technique guarantees a typical positive biopsy result in $60-70 \% .^{10)}$

On the other hand, the type and amount of fibrosis are of particular interest in the arrhythmogenic right ventricle. The working group of Thiene in Padua compared endomyocardial biopsies in heart transplant patients, dilated cardiomyopathy and arrhythmogenic right ventricular cardiomyopathy. ${ }^{3)}$ Transmural or subendocardial lipomatosis in more than $3 \%$ per biopsy and heavy fibrosis of more than $40 \%$ per biopsy were highly significant diagnostic criteria of arrhythmogenic right ventricular cardiomyopathy.

Mehta et $\mathrm{al}^{11)}$ and Wiesfeld et $\mathrm{al}^{4)}$ argued that different types of fibrosis in the same biopsy is a good marker of dysplasia / cardiomyopathy of the right ventricle.

The present results underline the importance of the sort and amount of fibrosis in arrhythmogenic right ventricular dysplasia diagnosed clinically by diagnostic criteria recently published. ${ }^{12)}$ Typical fibrolipomatosis was a very specific, but extremely insensitive finding in this cohort of patients. However, it has to be clearly pointed out that the histological definition of arrhythmogenic right ventricular dysplasia was based on lipomatosis, atrophy of the myocardium and a finer form of fibrosis surrounding individual myocytes or smaller groups of myocytes. Other features such as lipomatosis without fibrosis and without myocardial atrophy were defined as normal findings. ${ }^{13)}$

Cases with a clinical diagnosis of arrhythmogenic right ventricular dysplasia had a much higher degree of pathological findings, a much higher degree of different sorts of fibrosis per biopsy, a much higher amount of fibrosis with regard to the semi-quantitative analysis used in this study and the typical form of finer fibrosis surrounding individual myocytes or smaller groups of myocytes without lipomatosis.

Pathological histological findings were obtained in two cases among those with clinically defined idiopathic right ventricular outflow tract tachycardia, which developed the typical form of idiopathic dilated cardiomyopathy in the follow-up with echocardiographic and angiographic dilatation and functional worsening of the left ventricle and signs of left ventricular hypertrophy in standard ECG. In all other cases biopsy findings were normal or showed only slight interstitial fibrosis.

With regard to the standard biopsy taken from the right ventricular septum, 
outflow tract tachycardia and arrhythmogenic right ventricular dysplasia could be differentiated with high accuracy provided that a very distinct analysis of endomyocardial biopsy was conducted. The most important point is that the analysis of the biopsy cannot be computerized with exact assessment of the amount of lipomatosis or fibrosis as recommended by the working group of Thiene, ${ }^{3)}$ but mostly depends on the skill and experience of the pathologist who must have a very distinct look at the form of fibrosis which is possibly the key to the histological definition of arrhythmogenic right ventricular dysplasia.

The frequency of typical fibrolipomatosis as a highly specific but insensitive biopsy finding is low due to sampling error, progression of the disease from subepicardial to transmural regions and sparing of the right ventricular septum.

With a highly experienced pathologist endomyocardial angiography-directed biopsies from the right ventricular free wall cannot be generally recommended, inasmuch as this technique probably has a higher complication rate and some similar limitations due to disease spreading and sampling error.

\section{REFERENCES}

1. Fontaine $\mathrm{G}$, Fontaliron $\mathrm{F}$, Linares-Cuz E, et al. The arrhythmogenic right ventricle. In: Iwa $T$, Fontaine G, editors. Cardiac Arrhythmias: Recent Progress in Investigation and Management. New York, NY: Elsevier Science Publishers BV, 1988, 189-202.

2. Strain J. Adipose dysplasia of the right ventricle: is endomyocardial biopsy useful? Eur Hcart J 1989; 10 (Suppl. D): 84-8.

3. Angelini A, Thiene G, Boffa GM, et al. Endomyocardial biopsy in right ventricular cardiomyopathy. Int J Cardiol 1993; 40: 273-82.

4. Wiesfeld ACP, Grijns HJGM, Van Dijk RB, et al. Potential role for endomyocardial biopsy in the clinical characterization of patients with idiopathic ventricular fibrillation: Arrhythmogenic right ventricular dysplasia - an undervalued cause. Am Heart J 1994; 127: 1421-24.

5. Oakley CM. Amyloid heart disease and cardiomyopathies difficult to classify. In: Goodwin J, Olsen E, editors. Cardiomyopathies. Realisations and expectations. Springer Berlin-Heidelberg: 1993, 193-211.

6. Blake LM, Abbott JA, Alla SR, Higgins CB, Scheinman MM. Sensitivity and specificity of MRI for the diagnosis of right ventricular dysplasia versus right ventricular outflow tract tachycardia. Circulation 1994; 90: I-300 (abstract).

7. Marcus FL, Fontaine GH, Guiraudon G, Frank R, Laurenceau JL, Malergue $S$, et al. Right ventricular dysplasia. A report of 24 adult cases. Circulation 1982; 65:384-98.

8. Fontaine G, Guiraudon G, Frank R, et al. Stimulation studies and epicardial mapping in ventricular tachycardia: Study of mechanisms and selection for surgery. In: Kulbertus HE, editors. Reentrant arrhythmias. Lancaster: MTP, 1978, 334-50.

9. Thiene G, Nava A, Corrado D, et al. Right ventricular cardiomyopathy and sudden death in young people. N Engl J Med 1988; 318: 129-33.

10. Wichter $T$, Hindricks $G$, Lerch $H$, et al. Regional myocardial sympathetic dysinnervation in arrhythmogenic right ventricular cardiomyopathy. An analysis using 123-I-meta-iodobenzylguanidine scintigraphy. Circulation 1994; 89: 667-83.

11. Mehta D, McKenna WJ, Ward DE, et al. Significance of signal-averaged electrocardiography in relation to endomyocardial biopsy and ventricular stimulation studics in patients with ventricular tachycardia without clinically apparent heart disease. J Am Coll Cardiol 1989; 14: 372-78.

12. McKenna WJ, Thiene G, Nava A, et al. Diagnosis of arrhythmogenic right ventricular dysplasia / 
cardiomyopathy. Br Heart J 1994; $71: 215-8$.

13. Fontaliran F, Fontaine G, Fillette F, et al. Frontieres nosologiques de la dysplasia arhythmogene. Variations quantitatives du tissu adipeux ventriculaire droit normal. Arch Mal Coeur 1991; 84: 33-8. 